\title{
Efficacy of stereotactic aspiration in deep-seated and eloquent-region intracranial pyogenic abscesses
}

\author{
Xavier G. Kocherry, M.Ch., ${ }^{1}$ Thimappa Hegde, M.Ch., ${ }^{1}$ Kolluri V. R. Sastry, M.Ch., ${ }^{1}$ \\ Aaron MohanTy, M.Ch. ${ }^{1,2}$
}

${ }^{\prime}$ Department of Neurosurgery, National Institute of Mental Health and Neurosciences, Bangalore, India; and ${ }^{2}$ Division of Neurosurgery, University of Texas Medical Branch at Galveston, Texas

\begin{abstract}
Object. Intracranial abscesses located deep in the cerebral parenchyma and in eloquent regions are often difficult to manage surgically. The authors have attempted to analyze the efficacy of stereotactic aspiration in the management of these abscesses.

Methods. Cases involving 22 patients (including 9 children) with deep-seated and/or eloquent-region intracranial abscesses who underwent CT- or MR imaging-guided stereotactic aspiration between January 1995 and July 2001 were analyzed.

Results. A definite source of infection could be identified only in 9 of the cases. In 18 patients, the abscess was deep seated, whereas in the rest it was located in the eloquent cortex. Five patients had abscesses located in multiple sites. In 17 patients only 1 aspiration was required; in 5 others subsequent procedures were required. In the initial postaspiration CT, minor hemorrhage was noted in 3 patients not requiring further intervention. Antibiotics were administered for a period varying from 4 to 8 weeks following aspiration. An early recurrence (within 2 weeks of initial aspiration) was evident in 5 patients. All recurrent abscesses were reaspirated. In 2 patients new abscesses developed while the patients were still receiving antibiotic therapy. There were no late recurrences. In 1 patient ventriculitis developed, with subsequent hydrocephalus requiring a shunt insertion. Follow-up CT scans showed complete resolution of the abscess in all patients. There were no deaths.

Conclusions. Stereotactic aspiration is a useful management option for abscesses located in eloquent or inaccessible regions. Repeated aspiration should be considered in patients in whom the initial aspiration proves ineffective or partially effective. Complete resolution may require repeated stereotactic aspirations and continued antibiotic therapy. (DOI: $10.3171 /$ FOC/2008/24/6/E13)
\end{abstract}

KEY WORDS • abscess - intracranial pyogenic abscess • stereotactic aspiration

S INCE the introduction of CT scanning as the principal diagnostic investigation in neurosurgery, there has been a significant reduction in mortality and morbidity in association with brain abscesses. ${ }^{18,19}$ Abscesses located in the deep parenchyma and in eloquent regions of the cortex have been traditionally managed either with aspiration or with empiric broad-spectrum antibiotic therapy. With the advent of refined and user-friendly stereotactic frames, many of the deep-seated abscesses can be accurately localized and aspirated. In the present article, we reviewed our experience with 22 patients with deep-seated intracranial abscesses treated over a 7-year period and analyzed the outcomes. We also analyze the cases in which repeated aspirations were required due to lack of adequate resolution of the abscesses after initial aspiration.

Abbreviations used in this paper: $\mathrm{CT}=$ computed tomography; $\mathrm{MR}=$ magnetic resonance.

\section{Clinical Materials and Methods}

Twenty-two cases involving patients with intracranial pyogenic abscesses treated by stereotactic aspiration between January 1995 and July 2001 at the National Institute of Mental Health and Neurosciences in Bangalore, India, were retrospectively reviewed. The 22 patients underwent 24 procedures for treatment of their abscesses. In all 22 patients the abscesses were located either in deep and inaccessible regions of the cerebral parenchyma or in the eloquent cortex, necessitating stereotactic aspirations. Patients with abscesses of tuberculous or fungal etiology were excluded from the study.

After the diagnosis, all patients underwent CT or MR imaging localization of the lesion, performed with either a Brown Robert Wells or a Leksell frame. The adults underwent the procedure under local anesthesia. In children general anesthesia was induced. Of the 24 aspirations, 21 were CT guided and 3 were MR-guided. The center of the abscess cavity was used as the target. A twist-drill craniotomy was performed in all patients using a 3-mm diameter 
TABLE 1

Location of abscesses in 22 patients*

\begin{tabular}{lc}
\hline \hline \multicolumn{1}{c}{ Location } & No. of Abscesses \\
\hline frontal & 11 \\
parietal & 8 \\
temporal & 4 \\
occipital & 4 \\
thalamus & 7 \\
corpus callosum & 1 \\
basal ganglia & 3 \\
brainstem (pons) & 1 \\
\hline
\end{tabular}

* Some patients had multiple abscesses.

drill. The abscess cavity was entered using a 1-mm canula, and gentle aspiration was performed using a 2-ml syringe. Every attempt was made to aspirate the maximum amount of pus at the initial procedure. The procedure was terminated when the aspirate became blood tinged or no further aspiration could be performed. During the procedure, the larger abscesses were irrigated with gentamicin in saline (8 $\mathrm{mg} / \mathrm{ml}$ ). Upon completion of the procedure, the wound was closed with a single skin suture.

After the initial aspiration and collection of pus for culture, empiric antibiotic treatment was initiated, using a combination of 3 antibiotics administered intravenouslyeither chloramphenicol, ampicillin, and metronidazole or a third-generation cephalosporin (cefotaxime, ceftriaxone), an aminoglycoside, and metronidazole. A CT scan was performed 4-6 hours postaspiration to exclude any hemorrhagic complications. Neuroimaging studies were repeated initially at weekly intervals and subsequently at intervals of 2 weeks. Appropriate antibiotics, depending on the culture results, were continued for a minimum of 4 weeks or longer. For cases in which culture results revealed that the infecting organism was sensitive to multiple antibiotics, an oral antibiotic with good central nervous system penetration was preferred. Follow-up CT scans were performed at regular intervals to assess the response to treatment and the size of the residual abscess.
"Early recurrence" was defined as increase or lack of reduction in the abscess diameter demonstrated on follow-up CT within 2 weeks of initial aspiration. Recurrences occurring $\geq 2$ weeks after the initial aspiration were considered "late recurrence." Abscesses that developed in locations other than the one initially aspired while the patient was being treated with antibiotic therapy were considered "new abscesses." Recurrent abscesses were managed with repeated aspirations to evacuate the residual pus and with continuation of antibiotic treatment. The patients were followed up for $\geq 6$ months after the complete disappearance of the lesion to exclude any delayed recurrence.

\section{Results}

\section{Etiology and Location of Abscesses}

In $13(59 \%)$ of 22 patients, a definite source of infection could not be detected. In 6 patients the abscesses were secondary to cardiogenic causes. There were 2 cases of otogenic and 1 case of pulmogenic abscess. All of these 9 patients were receiving treatment with antibiotic therapy for their primary infection prior to the neurosurgical procedure. In addition, 6 others had been treated with antibiotic agents prior to the aspiration and obtaining of pus for culture.

Most of the abscesses occurred in the frontal lobe (11 abscesses), with the parietal lobe (8 abscesses) and the thalamus ( 7 cases) being the next most common sites (Table 1 ). There were 3 abscesses in the basal ganglia, and one each in the corpus callosum and pons (Fig. 1 left). Five patients had multiple abscesses in different locations. The larger abscesses with mass effect were treated with stereotactic aspiration while the smaller ones were medically managed. In the 17 patients with single abscesses, 11 abscesses were unilocular and 6 were multilocular. Ten abscesses were $11-20 \mathrm{~mm}$ in diameter, 9 were $21-30 \mathrm{~mm}$ in diameter, and only 4 were $\geq 31 \mathrm{~mm}$ in diameter. Only one abscess was $<10 \mathrm{~mm}$ in diameter. In 18 of the 24 abscesses, a reduction of size to $<5 \mathrm{~mm}$ was demonstrated in the postaspiration scan (Fig. 1 center).
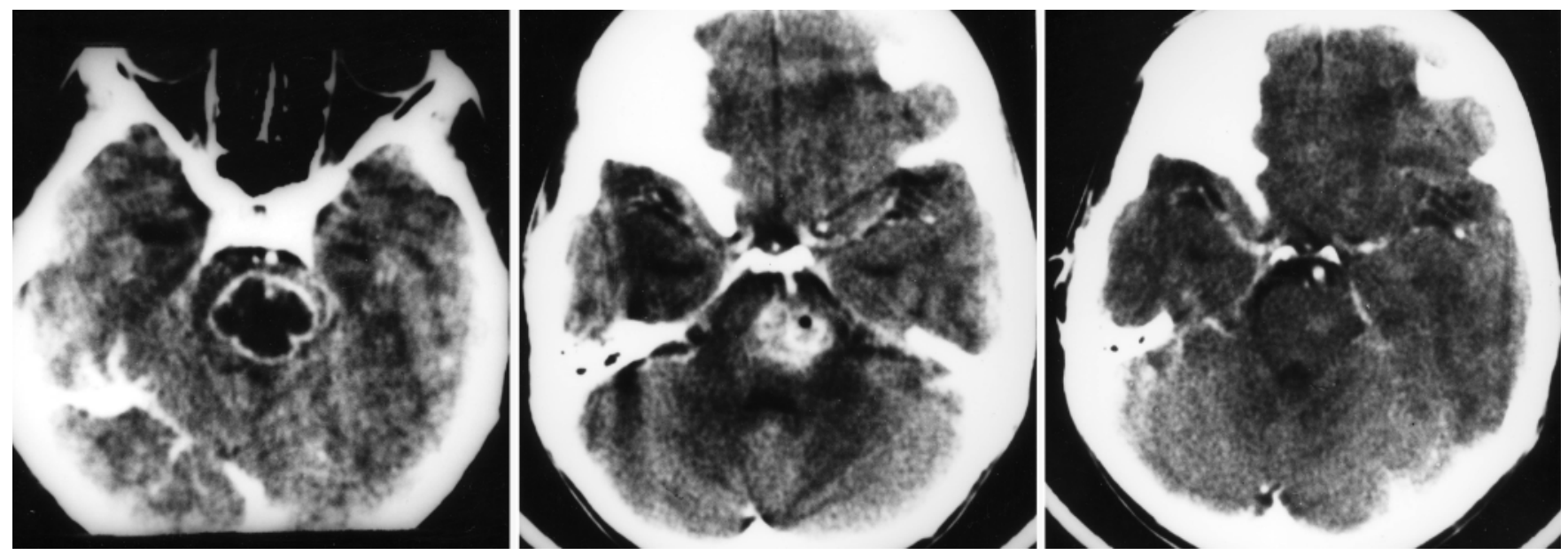

FIG. 1. Contrast-enhanced CT scans. Left: Pretreatment scan demonstrating a large brainstem abscess. Center: Scan obtained after stereotactic aspiration revealing significant reduction in abscess size. There is a small amount of bleeding in the abscess cavity around which the capsule is collapsed. Right: Follow-up scan obtained after 4 weeks of postaspiration antibiotic therapy revealing evidence of complete resolution with a small speck of enhancement. 


\section{Culture Results}

The initial culture yielded organisms in only 9 patients. In 6 of these, mixed flora consisting of both aerobes and anaerobes were isolated. The predominant anaerobes were Bacteroides species; whereas Streptococcus hemolyticus and nonhemolytic streptococci were the most commonly isolated aerobes.

\section{Postoperative Course}

In 3 patients, the postoperative CT scan demonstrated mild bleeding into the abscess cavity, but no surgical intervention was required for treatment of the bleeding. One patient developed ventriculitis in the postoperative period. He underwent repeated intraventricular instillation of antibiotics and subsequent placement of a ventriculoperitoneal shunt for persistent hydrocephalus.

\section{Recurrence of Abscesses}

The follow-up period varied from 6 months to 6 years. The minimum duration of follow-up was 6 months following complete resolution of the abscess (Fig 1 right).

Of the 22 patients, 5 had an early recurrence requiring a further procedure. Three of these 5 underwent repeated stereotactic aspiration (Tables 2 and 3). In 1 patient the abscess was reaspirated through the same drill hole using a freehand technique; and in 1 other patient bur-hole freehand aspiration was performed, as the abscess had increased in size during the follow-up period. In 4 of the 5 cases in which repeated aspiration was performed, the pus obtained at the second aspiration did not reveal any organisms, while in 1 the same organism was found as in the initial culture (nonhemolytic streptococci).

In 1 of the 5 early recurrences, the abscess was multilocular, while in the 4 others it was unilocular. In 4 of the 5 cases, the initial abscess diameter was $>31 \mathrm{~mm}$; in 1 it was $17 \mathrm{~mm}$. In all 5, the initial CT scans demonstrated that the abscesses had thick capsules, suggesting that they were in the chronic phase. The initial postaspiration CT showed a significant reduction (to $<5 \mathrm{~mm}$ ) in 2 of these, whereas in 3 others, the abscess size had reduced. The initial postaspiration $\mathrm{CT}$ showed a significant reduction (to $<5 \mathrm{~mm}$ ) in 2 of these, whereas in 3 others, the reduction was more modest (from $>31 \mathrm{~mm}$ to $11-20 \mathrm{~mm}$ ).

A late recurrence was not detected in any of the patients during the follow-up period. New lesions were detected in 2 patients, but the lesions were not considered for any surgical intervention because they were small and could be effectively managed with antibiotics.

\section{Discussion}

The ideal management of brain abscess aims at reducing the mass effect and resultant increased intracranial pressure with effective control of the infective process. Several management options have been described in the literature, including medical management with antibiotics, aspiration of pus, and excision of the abscess. ${ }^{22}$ Although antibiotic therapy is still the mainstay of treatment, pure medical management is usually reserved for the initial stages in which the abscess is not well formed. At most centers manage-
TABLE 2

Summary of abscess characteristics and procedures performed in 22 cases

\begin{tabular}{lc}
\hline \multicolumn{1}{c}{ Description } & No. of Abscesses \\
\hline cases involving abscesses in single locations & 17 \\
unilocular & 11 \\
multilocular & 6 \\
cases involving abscesses in multiple locations & 5 \\
abscesses requiring 1 surgical procedure & 24 \\
1 aspiration & 21 \\
2 aspirations* & 3 \\
abscesses requiring repeated surgical treatment & 5 \\
repeated stereotactic aspiration & 3 \\
freehand aspiration & 2 \\
\hline
\end{tabular}

* During the same surgical session.

ment would ideally include obtaining a sample of pus from the abscess to determine appropriate antibiotic therapy.

Aspiration of pus from the abscess cavity via a bur hole has been widely practiced and has been the mainstay of treatment. ${ }^{5,9,12,15,23}$ The location of the bur hole is carefully selected in order to minimize the brain tissue traversed during the procedure. Aspiration of pus, while relieving the increased intracranial pressure, provides pus for culture and sensitivity determination. It also permits irrigation of the abscess cavity and instillation of antibiotics in larger abscess cavities. Being relatively safe, it can be performed in critically ill and moribund patients under local anesthesia.

Alternatively, excision of the abscess via a craniotomy has been advocated as the treatment of choice for abscesses with well-formed capsules. ${ }^{3,20}$ Excision of the abscess has the advantage of facilitating removal of all the purulent material along with the wall of the abscess, while providing immediate relief of intracranial pressure and allowing a relatively shorter duration of antibiotic therapy. Occasionally initial aspiration and subsequent antibiotic therapy is followed by excision of the abscess wall (secondary excision). ${ }^{20,22}$

Aspiration of pus from the abscess cavity has been performed using freehand techniques or with ultrasound or stereotactic guidance. ${ }^{10,13,20,21}$ Stereotactic procedures have the advantage of localizing the abscess cavity with extreme precision, thus reducing the multiple attempts at aspiration witnessed with freehand techniques. This is of considerable importance in deep-seated abscesses and abscesses located in eloquent areas where accurate localization is considered essential. ${ }^{6-8,10,16}$

The present study extended over a 7-year period and only involved patients with brain abscesses treated with stereotactic aspiration. During the same period 176 intracranial abscesses were excised and 289 abscesses were aspirated without stereotactic guidance. Thus, the studied population represents a selected group of patients who were considered for stereotactic aspiration. We considered stereotactic aspiration only for patients with abscesses located in deep white matter, the basal ganglia, the thalamus or the brainstem, or abscesses located in eloquent cortex. Patients who had undergone freehand bur hole aspiration or craniotomy and excision were excluded from the study. This study did not include any cases of cerebellar abscess- 
X. G. Kocherry et al.

TABLE 3

Clinical characteristics of 5 cases of recurrent abscesses

\begin{tabular}{|c|c|c|c|c|c|c|c|c|}
\hline $\begin{array}{l}\text { Case } \\
\text { No. }\end{array}$ & Etiology & $\begin{array}{l}\text { Nature of } \\
\text { Abscess }\end{array}$ & $\begin{array}{l}\text { Initial Size } \\
\text { in } \mathrm{mm}\end{array}$ & $\begin{array}{l}\text { Abscess } \\
\text { Capsule }\end{array}$ & $\begin{array}{l}\text { Postaspiration } \\
\text { Size in mm }\end{array}$ & $\begin{array}{l}\text { Subsequent } \\
\text { Procedure }\end{array}$ & $\begin{array}{l}\text { Culture } \\
\text { Report }\end{array}$ & HIV Status \\
\hline 1 & idiopathic & unilocular & $>31$ & well defined & $<5$ & freehand aspiration & $\begin{array}{l}\text { nonhemolytic } \\
\text { streptococci }\end{array}$ & negative \\
\hline 2 & idiopathic & multilocular & $>31 *$ & well defined & $11-20$ & stereotactic aspiration & no growth & negative \\
\hline 3 & otogenic & unilocular & $>31$ & well defined & $11-20$ & freehand aspiration & no growth & negative \\
\hline 4 & idiopathic & unilocular & 17 & well defined & $<5$ & stereotactic aspiration & no growth & negative \\
\hline 5 & cardiogenic & unilocular & $>31$ & well defined & $11-20$ & stereotactic aspiration & no growth & negative \\
\hline
\end{tabular}

* Diameter of the largest loculation.

es as they have been preferably managed at our institution with craniotomy and excision.

\section{Mortality and Morbidity}

Such stereotactic procedures as needle biopsy and aspiration have been reported to have been associated with a very low morbidity and mortality. ${ }^{17}$ The incidence of hemorrhage in the probe track is considerably reduced as probes are blunt-tipped and the associated rigid fixation avoids the "leukotome effect" seen with a freehand aspiration. ${ }^{1}$ Though 3 of our patients experienced mild bleeding into the cyst cavity as demonstrated in routine postaspiration $\mathrm{CT}$, all were asymptomatic and none required a surgical procedure, indicating that these were relatively minor complications.

In our study, ventriculitis developed in 1 patient with a thalamic abscess. The ventriculitis was managed by intraventricular instillation of antibiotics along with intravenous antibiotic therapy, but the patient subsequently developed hydrocephalus requiring insertion of a ventriculoperitoneal shunt. Although none of our patients died during the study period, other authors have reported various mortality rates $(0-7 \%)$ in patients with brain abscesses treated with stereotactic aspirations. ${ }^{2,4,10,14}$

\section{Recurrence of Abscesses}

Recurrence of intracranial abscesses during treatment with antibiotic agents has been described in the literature. The reported rates of recurrences after stereotactic aspiration vary from 0 to $24 \%{ }^{2,4,10,14}$ In an early report, Dyste et al. ${ }^{6}$ reported $100 \%$ success with a single stereotactic procedure. In subsequent studies, however, recurrence has been reported following initial stereotactic aspiration. Kondziolka et al. ${ }^{10}$ reported a $72 \%$ success rate with single-procedure stereotactic management, with multiple procedures resulting in resolution in an additional $21 \%$ of patients. Mamelak et al. ${ }^{14}$ reported that $62 \%$ of their patients required additional surgery for further drainage after the initial aspiration. A reoperation was considered if an enlargement of the abscess was seen after a 2-week interval or no reduction in size was observed after 3-4 weeks of antibiotic therapy following the initial aspiration. In another study, Kutlay et al. ${ }^{11}$ noted a recurrence in $2(15.3 \%)$ of 13 patients who had had a documented reduction in abscess size after the initial aspiration and were being treated with antibiotic and hyperbaric oxygen therapy. The lesions did, however, resolve with repeated aspiration and continued antibiotic therapy.

In the present series, there were 5 early recurrences (rep- resenting $21 \%$ of our patient group). There was no late recurrence. All 4 patients who had an abscess diameter $\geq 3$ $\mathrm{cm}$ experienced recurrence, indicating that the initial size of the abscess is an important factor in the outcome of stereotactic aspiration. In a previous communication, patients cured with only medical therapy were reported to have had relatively smaller abscesses (average diameter $1.7 \mathrm{~cm}$ ) than those in whom medical therapy failed (average diameter $4.2 \mathrm{~cm}) .{ }^{18}$

Three of 5 recurrent abscesses in our patient group were deep seated, indicating that depth of the lesion was another significant factor for recurrence. Of these, one was in the basal ganglia and 2 others were in the thalamus. It has been reported that residual foci of infection in cortical gray matter could more easily be eradicated than residual foci in the periventricular regions because of the greater vascular supply of gray matter relative to white matter., ${ }^{6,24}$ The size of the postaspiration residual abscess was also related to recurrence. In 4 of the 5 patients who had recurrent abscesses in our case series, the postoperative CT performed within 6 hours of aspiration revealed a residual abscess size between 11 and $20 \mathrm{~mm}$, indicating that inadequate aspiration at the initial session was a possible factor for recurrence. A similar view has been expressed by Kondziolka et al. ${ }^{10}$

Long-term immune suppression has been considered as a factor associated with recurrence,,$^{10}$ but there were no patients receiving long-term immunosuppressive therapy in our patient group. Similarly, we did not encounter any cases in which recurrence was associated with the presence of antibiotic-resistant organisms.

Repeated stereotactic aspiration or craniotomy and excision (secondary excision) have been recommended for the surgical management of recurrent abscesses. ${ }^{14,20}$ We did not consider secondary excision in view of the abscess locations and preferred to manage them with repeated aspirations and continued antibiotic therapy. Though we prefer stereotactic techniques for reaspiration, in 2 patients we used the previous twist-drill craniotomy with the depth calculated from the CT scan for a freehand aspiration to reach the abscess cavity. This technique was not associated with any untoward effects considering that it is almost impossible to deviate through the twist-drill hole craniotomy track while coursing through the calvaria if the probe is of a similar diameter as the drill bit. With the present strategy we achieved complete resolution in all cases, with no late recurrence.

In a unique report, Kutlay et al. ${ }^{11}$ described using adjuvant hyperbaric oxygen along with stereotactic aspiration and systemic antibiotic therapy and achieving a $15 \%$ recur- 
rence rate in 13 patients with intracranial abscesses. The authors opined that hyperbaric oxygen therapy can reduce the duration of antibiotic therapy and the need for reoperation. Stereotactic placement of an intracavitary catheter for evacuation of pus and intermittent instillation of antibiotics also has been described as a means of reducing the risk of recurrence after aspiration. ${ }^{4}$

\section{Conclusions}

Stereotactic aspiration of deep-seated abscesses and eloquent-region lesions is safe and can be performed in all age groups. Early recurrence was seen in $20 \%$ of the patients in the present series. The recurrent abscesses could be satisfactorily treated with a combination of antibiotics and repeated aspiration. There were no late recurrences with this modality of management.

\section{References}

1. Arunkumar MJ, Rajshekhar V, Chandy MJ, Thomas PP, Jacob CK: Management of brain abscesses in renal transplant recipients. Postgrad Med J 76:207-211, 2001

2. Barlas O, Sencer A, Erkan K, Kraksoy H, Sencer S, Bayindir C: Stereotactic surgery in the management of brain abscess. Surg Neurol 52:404-411, 1999

3. Brewer NS, MacCarty CS, Wellman WE: Brain abscess: a review of recent experience. Ann Intern Med 82:571-576, 1975

4. Broggi G, Franzini A, Peluchetti P, Servello P: Treatment of deep brain abscesses by stereotactic implantation of an intracavitary device for evacuation and local application of antibiotics. Acta Neurochir (Wien) 76:94-98, 1985

5. Chacko, AC, Chandy MJ: Diagnostic and staged stereotactic aspiration of multiple bihemispheric pyogenic brain abscess. Surg Neurol 48:278-283, 1997

6. Dyste GN, Hitchon PW, Menezes AH, VanGilder JC, Greene GM: Stereotaxic surgery in the treatment of multiple brain abscesses. J Neurosurg 69:188-194, 1988

7. Hasdemir MG, Ebeling U: CT-guided stereotactic aspiration and treatment of brain abscesses. An experience with 24 cases. Acta Neurochir (Wien) 125:58-63, 1993

8. Itakura T, Yokote H, Ozaki F, Itatani K, Hayashi S, Komai N: Stereotactic operation for brain abscess. Surg Neurol 28:196200, 1987

9. Kagawa M, Takeshita M, Yato S, Kitamura K: Brain abscess in congenital cyanotic heart disease. J Neurosurg 58:913-917, 1983

10. Kondziolka D, Duma CM, Lunsford LD: Factors that enhance the likelihood of successful stereotactic treatment of brain abscess. Acta Neurochir (Wien) 127:85-90, 1994

11. Kutlay M, Colak A, Yildiz S, Demircan N, Akin ON: Stereotactic aspiration and antibiotic treatment combined with hyperbaric oxygen therapy in the management of bacterial brain abscesses. Neurosurgery 57:1140-1146, 2005

12. Law JD, Lehman RA, Kirsch WM, Ehni G: Diagnosis and treatment of abscess of the central ganglia. J Neurosurg 44:226-232, 1976

13. Lunsford LD: Stereotactic drainage of brain abscess. Neurol Res 9:270-274, 1987

14. Mamelak A, Mampalam T, Obana W, Rosenblum ML: Improved management of multiple brain abscesses: a combined surgical and medical approach. Neurosurgery 36:76-86, 1995

15. Matson DD, Salam M: Brain abscess in congenital heart disease. Pediatrics 27:772-789, 1961

16. Rajshekhar V, Chandy MJ: Successful stereotactic management of a large cardiogenic brain stem abscess. Neurosurgery 34:368371, 1994

17. Ranjan A, Rajsekhar V, Joseph T, Chandy MJ, Chandi SM: Nondiagnostic CT-guided stereotactic biopsies in a series of 407 cases: influence of CT morphology and operator experience. J Neurosurg 79:839-844, 1993

18. Rosenblum ML, Hoff JT, Norman D, Weinstein PR, Pitts L: Decreased mortality from brain abscesses since advent of computerized tomography. J Neurosurg 49:658-668, 1978

19. Rousseaux M, Lesoin F, Destee A, Jomin M, Petit H: Developments in the treatment and prognosis of multiple cerebral abscesses. Neurosurgery 16:304-308, 1985

20. Sharma SS, Gupta SK, Khosla VK: Current concepts in management of pyogenic brain abscess. Neurol India 48:105-111, 2000

21. Stapleton SR, Bell BA, Uttley D: Stereotactic aspiration of brain abscesses: is this the treatment of choice? Acta Neurochir (Wien) 121: 15-19, 1993

22. Stephanov S: Surgical treatment of brain abscess. Neurosurgery 22:724-730, 1988

23. Tekkök IH, Erbengi A: Management of brain abscess in children: review of 130 cases over a period of 21 years. Childs Nerv Syst 8:411-416, 1992

24. Whelan MA, Hilal SK: Computed tomography as a guide in the diagnosis and follow-up of brain abscesses. Radiology 135: $663-571,1980$

Manuscript submitted February 1, 2008.

Accepted March 19, 2008.

Address correspondence to: Aaron Mohanty, M.Ch., Division of Neurosurgery, University of Texas Medical Branch at Galveston, John Sealy Annex, Room 9.208, 301 University Boulevard, Galveston, Texas 77555-0517. email: aarmohanty@yahoo.com. 